Cite this: J. Mater. Chem. A, 2014, 2 , 6879

Received 17th January 2014 Accepted 18th February 2014

DOI: $10.1039 / c 4 t a 00285 g$

www.rsc.org/MaterialsA

\title{
Rapid phosphine-free synthesis of CdSe quantum dots: promoting the generation of Se precursors using a radical initiator $\uparrow$
}

\author{
Bo Hou, ${ }^{a}$ David Benito-Alifonso, ${ }^{a}$ Richard Webster, ${ }^{b}$ David Cherns, ${ }^{b}$ \\ M. Carmen Galan ${ }^{a}$ and David J. Fermín ${ }^{\star a}$
}

\begin{abstract}
The replacement of phosphine containing compounds in the synthesis of II-VI quantum dots (QDs) via the 'hot-injection' method has received considerable attention in recent years, in particular toward scaling-up production. A key bottleneck in current approaches is the poor solubility of elemental Se in solvents such as 1-octadecene (1-ODE) or oleylamine requiring a heating temperature of $200{ }^{\circ} \mathrm{C}$ for several hours, or the introduction of additives such as alkylthiols or $\mathrm{NaBH}_{4}$, or replacement by $\mathrm{SeO}_{2}$. In the present work, we elucidate the fundamental steps in the dissolution of elemental Se in 1-ODE and oleylamine with the view of facilitating the large-scale synthesis of CdSe QDs. The main organoselenium species generated during the solubilisation of elemental Se in 1-ODE and oleylamine were identified by $1 \mathrm{D}$ and 2D NMR spectroscopy $\left({ }^{1} \mathrm{H},{ }^{13} \mathrm{C}\right.$ and $\left.{ }^{77} \mathrm{Se}\right)$. Experimental evidence suggests that the rate determining step is the formation of Se radicals, via homolytic cleavage of the Se-Se bond, that attack the allylic proton in 1ODE and oleylamine. Plausible reaction pathways in both systems are proposed. Finally, we demonstrate that the radical-mediated solubilisation of Se can be significantly accelerated by the addition of azobisisobutyronitrile (AIBN), a common radical initiator used in the polymer industry. In this way, a highly concentrated Se precursor was prepared. The "hot-injection" of the Se precursor into CdO containing 1-ODE solution leads to the formation of highly luminescent CdSe QDs with a well-defined cubic structure.
\end{abstract}

\section{Introduction}

The optical properties of CdSe quantum dots (QDs) have generated a tremendous amount of interest due to their potential exploitation in a variety of fields including lightemitting devices, ${ }^{1}$ imaging probes ${ }^{2}$ and absorber layers in photovoltaic devices. ${ }^{3}$ Colloidal synthesis is widely recognized as key for large-scale fabrication of highly monodispersed and stable QDs. The seminal work by Murray et al. ${ }^{4}$ introduced the methodology of injecting phosphine-chalcogenide precursors into an organometallic solution at temperatures above $300^{\circ} \mathrm{C} .{ }^{4 b}$ Another important contribution in the field was made by Peng and co-workers, replacing the highly unstable organometallic precursors by metal oxides (e.g. CdO) in the presence of stearic acid. ${ }^{5}$ With regard to the solubilization of the chalcogenide component, Lewis bases such as tributylphosphine (TBP) or trioctylphosphine (TOP)

${ }^{a}$ School of Chemistry, University of Bristol, Cantocks Close, Bristol BS8 1TS, UK; Web: http://www.chm.bris.ac.uk/pt/electrochemistry.E-mail: David.Fermin@bristol.ac.uk; Tel: +44 (0)1179288981

${ }^{b}$ School of Physics, University of Bristol, Tyndall Avenue, Bristol BS8 1TL, UK

$\uparrow$ Electronic supplementary information (ESI) available. See DOI: $10.1039 / \mathrm{c} 4$ ta00285g have been extensively used. ${ }^{6}$ However, these phosphines are highly flammable and corrosive, ${ }^{7}$ prompting the search for alternative approaches.

Mulvaney and co-workers reported the first phosphine-free synthesis of CdSe, based on the dissolution of Se in 1-octadecene (1-ODE) after prolonged periods of heating at high temperatures. ${ }^{8}$ Subsequently, other research groups have employed similar approaches to synthesize phosphine-free nanocrystals. ${ }^{9}$ Solvents such as olive oil, ${ }^{10}$ paraffin, ${ }^{11}$ and oleylamine ${ }^{12}$ have also been implemented. In addition to long time heating protocols aimed at facilitating the dissolution of Se, additives including $\mathrm{NaBH}_{4}{ }^{13}$ and alkylthiols ${ }^{14}$ have been introduced. The disadvantage of the latter approaches is the introduction of additional elements (e.g. Na, B, and S) which could appear as impurities in the final product. Recent studies have demonstrated that the synthesis of QDs can be carried out by employing insolubilized $\mathrm{Se}^{15}$ or $\mathrm{SeO}_{2}{ }^{16}$ powder with good yields. In these approaches, the reported Se concentration in precursor solution is not higher than $0.2 \mathrm{~mol} \mathrm{dm}^{-3}$, which represents a bottleneck towards scale-up. In order to make the reaction suitable to the industrial scope, a good understanding of the Se dissolution process is crucial. Raston and co-workers proposed that Se-Se and Se-C bonding compounds are the main species after the dissolution of Se into 1-ODE. ${ }^{17}$ However, the 
mechanism of formation and nature of the Se precursors in these solvents remain to be fully elucidated.

In this work, we investigate the process involved in the dissolution of Se in 1-ODE and oleylamine by NMR, FTIR and mass spectroscopy, with the aim of optimizing this key step which is considered the bottleneck in the phosphine-free synthesis of CdSe QDs. ${ }^{12 b, 14}$ Our results confirm that the process is initiated by the attack of thermally generated Se radicals to the allylic position of 1-ODE upon heating at temperatures above $200{ }^{\circ} \mathrm{C}$, in agreement with previous studies. ${ }^{17}$ We show that this reaction manifests itself by the migration of the terminal olefin group in 1-ODE and cis/trans isomerisation in oleylamine, generating a range of organoselenium structures. Furthermore, we show for the first time that Se dissolution in 1-ODE can be significantly accelerated by addition of trace amounts of the radical initiator azobisisobutyronitrile (AIBN, I). This approach allows not only the preparation of Se precursor solutions with concentrations as high as $1.0 \mathrm{~mol} \mathrm{dm}^{-3}$, but also the facile synthesis of highly luminescent CdSe QDs under phosphine-free conditions yielding phase-pure monodispersed cubic nanocrystals.

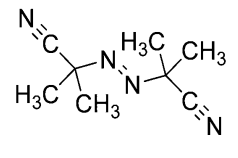

I

\section{Experimental}

\subsection{Se dissolution in 1-ODE and oleylamine}

The dissolution of elemental Se (3 mmol) into 1-ODE (9 mmol, $2.88 \mathrm{~cm}^{3}$ ) or oleylamine ( $9 \mathrm{mmol}, 4.23 \mathrm{~cm}^{3}$ ) was investigated by taking aliquots of the reaction mixture during the following heating program: room temperature (step I), $60{ }^{\circ} \mathrm{C}$ for $30 \mathrm{~min}$ under vacuum (step II), $130^{\circ} \mathrm{C}$ for 30 min under vacuum (step III), $200{ }^{\circ} \mathrm{C}$ for $30 \mathrm{~min}$ (step IV) and $20 \mathrm{~h}$ (step V) under Ar. Each aliquot was analysed by $1 \mathrm{D}^{1} \mathrm{H},{ }^{13} \mathrm{C}$ and $2 \mathrm{D}$ NMR, as well as FTIR spectroscopy. The concentration of the final Se solution in 1-ODE or oleylamine is 1.04 and $0.71 \mathrm{~mol} \mathrm{dm}^{-3}$, respectively.

\subsection{Facile Se dissolution promoted by AIBN}

Following a similar procedure as in the previous experiment, a mixture of Se and 1-ODE $\left(1.04 \mathrm{~mol} \mathrm{dm}^{-3}\right)$ was prepared with the addition of $0.6 \mathrm{mg}$ of AIBN at room temperature. The composition of the solution was investigated at room temperature under $\mathrm{Ar}$ (step I) and after heating at $200^{\circ} \mathrm{C}$ for $1 \mathrm{~h}$ (step II), $2 \mathrm{~h}$ (step III) and $3 \mathrm{~h}$ (step IV).

\subsection{Phosphine-free synthesis of CdSe QDs}

The QDs were prepared based on the method reported by Peng ${ }^{18}$ with a commonly employed Se precursor concentration $(0.1 \mathrm{~mol}$ $\left.\mathrm{dm}^{-3}\right)$. Typically, Se $(0.0395 \mathrm{~g})$ and 1-ODE $\left(5 \mathrm{~cm}^{3}\right)$ are loaded into a $50 \mathrm{~cm}^{3}$ two-neck flask and heated at $60{ }^{\circ} \mathrm{C}$ under vacuum for $1 \mathrm{~h}$, followed by addition of $0.001 \mathrm{~g}$ of AIBN $\left(1 \% \mathrm{~mol} \mathrm{~mol}^{-1}\right)$. The temperature is subsequently increased to $200{ }^{\circ} \mathrm{C}$ for $1 \mathrm{~h}$ under Ar, leading to the dissolution of Se (clear yellow solution). $\mathrm{CdO}(0.0254 \mathrm{~g})$, stearic acid $(0.228 \mathrm{~g})$ and 1-ODE $\left(20 \mathrm{~cm}^{3}\right)$ were introduced into a $250 \mathrm{ml}$ three-neck round bottom flask and dried in a vacuum at $100{ }^{\circ} \mathrm{C}$ for $30 \mathrm{~min}$. The metal-precursor solution was subsequently heated to $320{ }^{\circ} \mathrm{C}$ under an $\mathrm{Ar}$ atmosphere. Once the solution became colourless, the Se precursor was quickly injected into the cadmium solution. The particles were allowed to grow for different times at $320^{\circ} \mathrm{C}$, after which the heating mantle was removed. The QDs were purified by dissolving in a methanol-hexane mixture $(\mathrm{v} / \mathrm{v}=3: 1)$ and centrifuged for $10 \mathrm{~min}$ to remove 1 -ODE, followed by addition of acetone and further centrifugation. The precipitate was dissolved in chloroform for further characterization.

\subsection{NMR, FTIR and electron microscopy methodologies and instrumentation}

Reactions requiring anhydrous conditions were performed under an atmosphere of either anhydrous nitrogen or argon. All glassware was flame-dried prior to use and glass syringes and needles were placed in an oven $\left(150{ }^{\circ} \mathrm{C}\right)$ for at least $2 \mathrm{~h}$ and allowed to cool in desiccators under an atmosphere of anhydrous nitrogen. Aliquots from the reaction mixture were collected at different stages of the heating program under an $\mathrm{Ar}$ atmosphere and samples were allowed to cool to room temperature before analysis. 1D, 2D ${ }^{1} \mathrm{H}$ NMR and ${ }^{13} \mathrm{C}$ NMR spectra from 2D proton correlation spectroscopy (COSY), carbon-proton heteronuclear single quantum correlation (HSQC) and carbon-proton heteronuclear multiple-bond correlation spectroscopy (HMBC) were recorded in a $400 \mathrm{MHz}$ Varian INOVA 400 instrument. Chemical shifts are quoted in parts per million (ppm) and referenced to $\mathrm{SiMe}_{4}\left({ }^{1} \mathrm{H}\right.$ NMR 0 ppm) and $\mathrm{CDCl}_{3}\left({ }^{13} \mathrm{C}\right.$ NMR $\left.77.16 \mathrm{ppm}\right)$. The ${ }^{13} \mathrm{C}$ NMR sequence parameters were set up as follows: a $45^{\circ}$ pulse of 7.70 $\mu \mathrm{s}, \tau_{2}=1.0 \mathrm{~s}$, and an acquisition time of $1.28 \mathrm{~s}$. The $1 \mathrm{D}$ and $2 \mathrm{D}$ ${ }^{77} \mathrm{Se}$ NMR spectra including $1 \mathrm{D}$ proton decoupled ${ }^{77} \mathrm{Se}$ NMR and 2D proton-selenium HMBC were recorded in a $500 \mathrm{MHz}$ Varian INOVA 500 instrument. The ${ }^{77} \mathrm{Se}$ NMR sequence parameters were set up as follows: $45^{\circ}$ pulse, $\tau_{2}=1.0 \mathrm{~s}$, and an acquisition time of $0.860 \mathrm{~s}$. A saturated solution of diphenyldiselenide in $\mathrm{CDCl}_{3}$ was used as an external standard. ${ }^{19}$ FTIR spectra were obtained using a Perkin-Elmer Spectrum 100 FTIR Spectrometer. The structure of the CdSe dots was investigated by powder XRD (BRUKER D8, Cu K $\alpha$ radiation $\lambda=1.54 \AA$ A) and JEOL 2011 high resolution TEM operating at $200 \mathrm{kV}$ fitted with an EDX Oxford Instruments ISIS 300 system. Selected area electron diffraction (SAED) analysis was performed using a Philips EM430 instrument with a camera length set at $270 \mathrm{~mm}$ and the electron wavelength at $0.251 \AA$.

\subsection{Electrospray ionization (ESI) and negative ion matrix assisted laser desorption ionization time-of-flight (MALDI- TOF) mass spectrometry (MS) analysis of Se-containing product}

In the 1-ODE system, the Se containing products were isolated by silica gel flash column chromatography using hexane as the eluent. In the oleylamine system, the Se containing products are 
isolated through vacuum distillation using a Kugerlrohr apparatus. The isolated products were dried under vacuum and re-dissolved into dichloromethane for $\mathrm{ESI}^{+}$analysis. MALDI analyses were recorded using a HP MALDI instrument (Applied Biosystems 4700 Proteomics Analyzer) using gentisic acid as the matrix.

\section{Results and discussion}

\subsection{Analysis of the Se precursor in 1-ODE}

Fig. 1 displays NMR and FTIR spectra of the Se-1-ODE mixture at various heating steps. ${ }^{13} \mathrm{C}$ NMR spectra show the emergence of new signals at $\delta=124.4$ and 131.7 ppm (Fig. 1a) after heating at $200{ }^{\circ} \mathrm{C}$ for $20 \mathrm{~h}$ under an Ar atmosphere (step V). ${ }^{1} \mathrm{H}$ NMR data also revealed the appearance of a new multiplet signal at $\delta=$ $5.42 \mathrm{ppm}$ at step V (Fig. 1b). The appearance of these signals occurs only after the complete dissolution of Se in 1-ODE. A comparison of the 2D HSQC NMR spectra of the solutions at steps I (Fig. 1c) and V (Fig. 1d) reveals the appearance of cis and trans carbon-proton correlations in the latter step, suggesting the migration of the terminal olefin moiety to form 2-octadecene (2-ODE). This analysis is supported by the 2D COSY NMR spectrum of sample $\mathrm{V}$ showing that the olefinic protons $(\delta=$ 5.41 and $5.43 \mathrm{ppm}$ ) are correlated with protons found at $\delta=1.63$ and $1.94 \mathrm{ppm}$ (see ESI [S3] $\dagger$ ), while the HSQC spectrum in Fig. $\mathrm{S} 4 \uparrow$ indicates that the protons at $\delta=1.63$ and $1.94 \mathrm{ppm}$ are bonded with carbon atoms at $\delta=18.0$ and $32.7 \mathrm{ppm}$, which are attributed to $\mathrm{CH}_{2}$ and $\mathrm{CH}_{3}$ moieties. Furthermore, the decrease

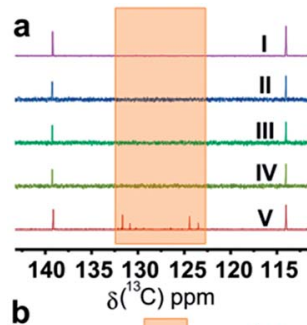

b
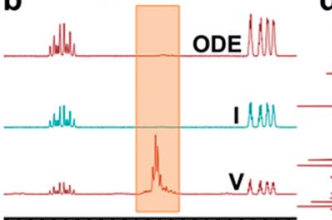

$\begin{array}{llllllll}5.9 & 5.7 & 5.5 & 5.3 & 5.1 & 4.9\end{array}$ $\delta\left({ }^{1} \mathrm{H}\right) \mathrm{ppm}$ c
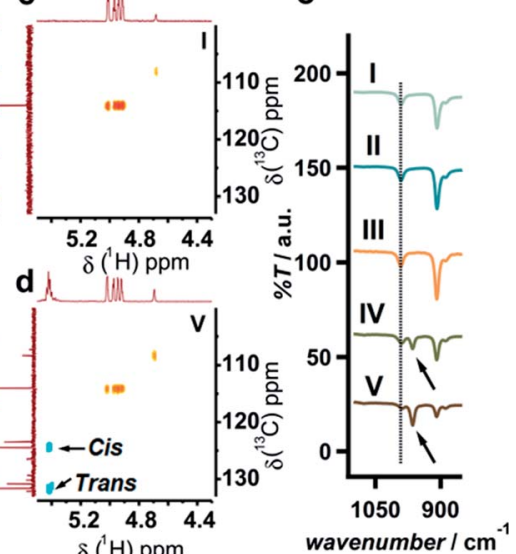

Fig. $1{ }^{13} \mathrm{C}$-NMR analysis of Se in 1-ODE at room temperature (step I), $60{ }^{\circ} \mathrm{C}$ for $30 \mathrm{~min}$ under vacuum (step II), $130{ }^{\circ} \mathrm{C}$ for $30 \mathrm{~min}$ under vacuum (step III), $200^{\circ} \mathrm{C}$ for $30 \mathrm{~min}$ (step IV) and $20 \mathrm{~h}$ (step V) under Ar (a). ${ }^{1} \mathrm{H}$-NMR spectra of the solution precursor at steps I and $\mathrm{V}$, as well as of $1-\mathrm{ODE}$ after heating at $200^{\circ} \mathrm{C}$ for $20 \mathrm{~h}$ under $\mathrm{Ar}$ in the absence of Se (b). The highlighted areas in (a) and (b) correspond to the signal associated with the olefin functional group. HSQC 2D NMR spectra of Se containing solutions at step I (c) and step V (d). The arrows indicate the correlation between ${ }^{13} \mathrm{C}-{ }^{1} \mathrm{H}$ from cis and trans olefin moieties. FTIR analysis of the precursor solutions at steps I to $\mathrm{V}$ (e). The arrows indicate a vibrational mode associated with the olefin moiety in trans 2-octadecene, while the dotted line indicates the position of the equivalent vibrational model in 1-ODE. of the IR bands at $\nu=991$ and $908 \mathrm{~cm}^{-1}$ and the emergence of a band at $\nu=964 \mathrm{~cm}^{-1}$ (Fig. 1e) are also consistent with the formation of 2-ODE.

Fig. 2 shows the ${ }^{77}$ Se NMR analysis of the Se precursor at step $\mathrm{V}$ after purification by chromatography. The NMR signals with chemical shifts of 416.4 (singlet), 373.1 (singlet), 373.3 (singlet), 307.4 (singlet) and $259.2 \mathrm{ppm}$ (singlet) are consistent with dialkyl polyselenides species. ${ }^{20}{ }^{1} \mathrm{H},{ }^{13} \mathrm{C},{ }^{77} \mathrm{Se} 1 \mathrm{D}$ and $2 \mathrm{D}$ NMR analyses (Fig. 3 and ESI $[\mathrm{S} 4] \dagger$ ) confirmed the formation of a

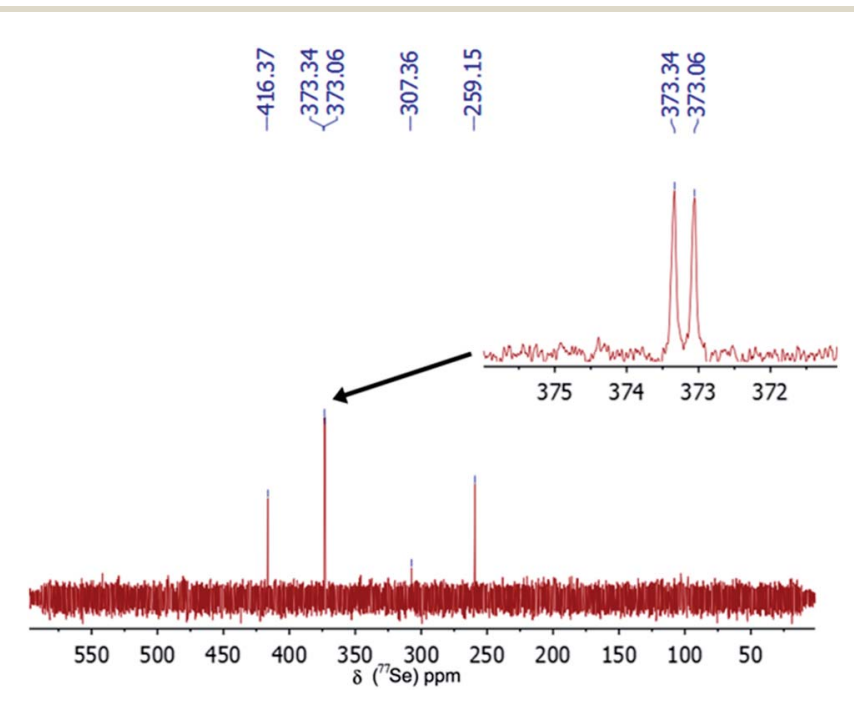

Fig. 2 Proton decoupled ${ }^{77} \mathrm{Se}$ NMR spectrum of the purified Se intermediate generated after dissolution of $1-\mathrm{ODE}$ after $20 \mathrm{~h}$ at $200^{\circ} \mathrm{C}$ under Ar. The inset shows two closed peaks with chemical shifts of 373.3 and $373.1 \mathrm{ppm}$.

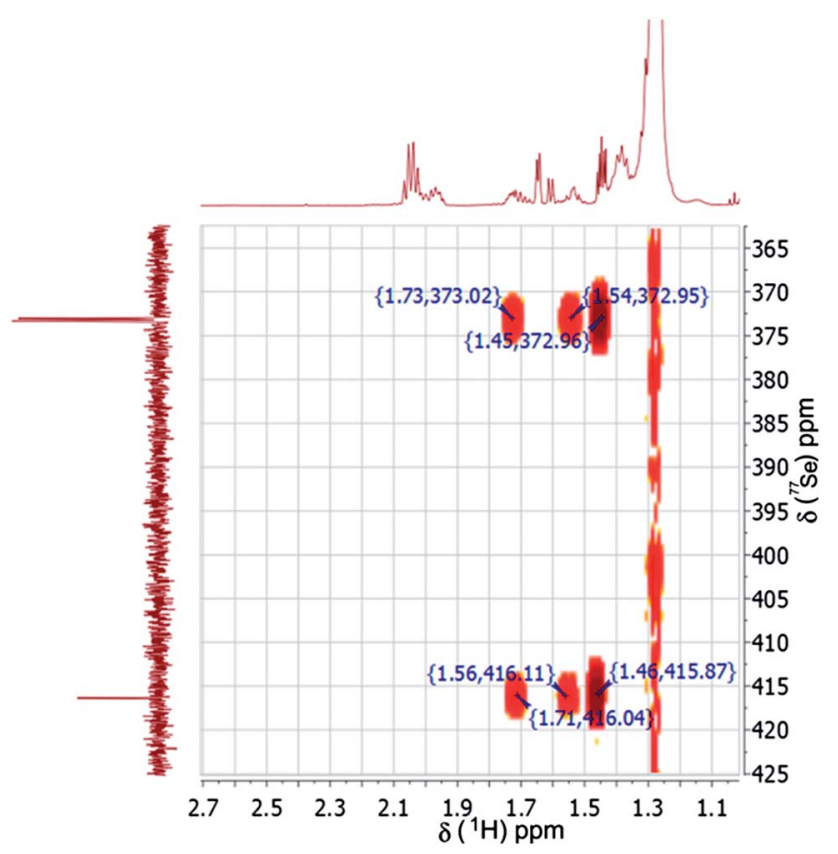

Fig. $3{ }^{1} \mathrm{H}$ and ${ }^{77} \mathrm{Se} \mathrm{HMBC} \mathrm{NMR}$ analysis of Se containing species. As indicated in the image, ${ }^{77} \mathrm{Se}$ NMR signals at $\delta=373.3$ and $373.1 \mathrm{ppm}$ correlate with the ${ }^{1} \mathrm{H}$ multiplet at $\delta=1.73$ and $1.54 \mathrm{ppm}$. 
carbon-selenium bond during the solubilisation process. The species formed are a mixture of structural isomers with similar properties, complicating their isolation. A thorough study of the mixture shows, as a common feature, the presence of an olefinic group alpha to the $C-S e$ bond.

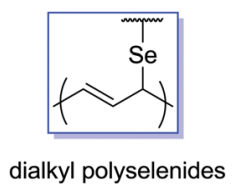

II

Fig. 3 shows the $2 \mathrm{D}{ }^{1} \mathrm{H}$ and ${ }^{77}$ Se HMBC NMR spectra of Se containing species, confirming the se bonding to a secondary carbon. The $2 \mathrm{D}^{1} \mathrm{H}$ and ${ }^{13} \mathrm{C}$ HMBC NMR spectra in Fig. S5 and S6 (see ESI [S4] $\dagger$ ) suggest that the secondary carbon bound to Se is next to an olefinic carbon and a saturated carbon. The $2 \mathrm{D}{ }^{1} \mathrm{H}$ and ${ }^{13} \mathrm{C}$ HSQC NMR spectra in Fig. S7 (see ESI [S4]†) further confirm that the olefinic moiety is vicinal to the $\mathrm{C}-\mathrm{Se}$ bond and (II) is proposed to be the general core structure.

Mass values collected from ESI and MALDI MS analyses suggested the species (III) as plausible structures being formed. Detail mass spectra can be found in the ESI (S1 and S2). $\dagger$ A correlation of NMR and mass spectrometry analysis results is listed in Table S1. $\dagger$

Based on the analysis of the NMR, FTIR, and MS data shown in Fig. 1-3 and Table S1, $\uparrow$ the dissolution of Se in 1-ODE can be rationalized in terms of a radical process thermally initiated by the homolytic cleavage of the Se-Se bond as highlighted in Scheme 1. The key step is the attack of the allylic proton in 1-ODE by Se radicals leading to the migration of the double bond and subsequent $\mathrm{H}$ transfer from the Se intermediate species. ${ }^{\mathbf{1 7 2 1}}$ This is further supported by subsequent experiments where no isomerization is observed upon extensive heating of 1-ODE in the absence of Se (shown in Fig. $1 \mathrm{~b}$ and also ESI [S5] $\dagger$ ). Moreover, no dissolution of Se takes place under similar conditions if 1-ODE is replaced by octadecane, which lacks the alkene functionality.

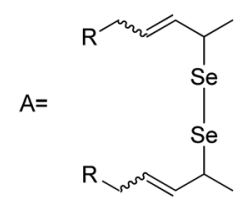

1,3-di(octadec-3-en-2-yl)diselenide

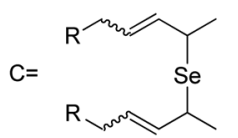

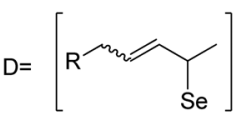

1,3-di(octadec-3-en-2-yl)selenide

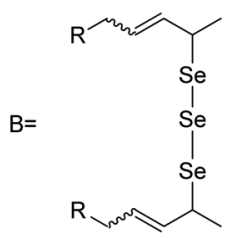

1,3-di(octadec-3-en-2-yl)triselenide

$\mathrm{R}=$

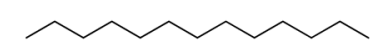

III

$$
\mathrm{Se}_{\mathrm{n}}-\mathrm{Se}+\mathrm{Se}_{\mathrm{n}}-\mathrm{Se}-\mathrm{Se}-\mathrm{Se}_{\mathrm{H}} \longrightarrow 2 \mathrm{Se}_{\mathrm{H}}-\mathrm{Se}
$$

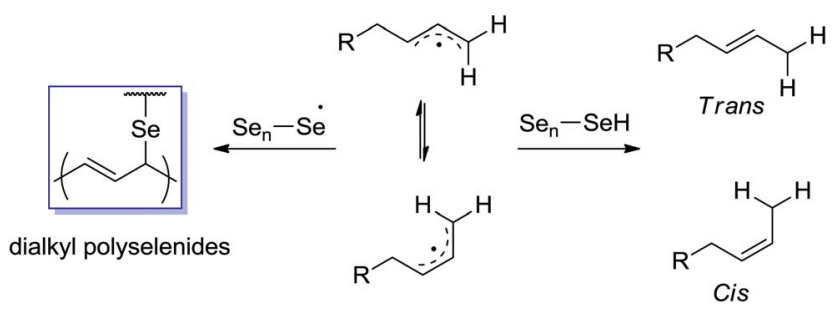

Scheme 1 Proposed mechanism for the thermally induced Se radical attack of the allylic proton in 1-ODE, leading to the dissolution of Se and the migration of the terminal olefin. Highlighted in the blue frame is the olefinic group alpha to the $\mathrm{C}-\mathrm{Se}$ bond which is a common feature present in the organoselenium species.

\subsection{Dissolution of Se into oleylamine}

In addition to 1-ODE, oleylamine is another solvent often used for the preparation of Se precursors. ${ }^{12 a}$ In our recent work, organoselenium species were detected by employing mass spectroscopy and ${ }^{1} \mathrm{H}$ and ${ }^{13} \mathrm{C}$ NMR spectroscopy, during the Se dissolution in oleylamine. ${ }^{22}$ Although isomerization of the double bond was identified in this instance, the depletion of the olefin moiety was also observed, which did not occur in the case of 1-ODE. ${ }^{22}$ Fig. 4 shows the HSQC NMR analysis of Se containing species at steps I and IV. A significant decrease in the signals at ${ }^{13} \mathrm{C} \delta=130 \mathrm{ppm}$ and ${ }^{1} \mathrm{H} \delta=5.35$ ppm upon heating at $200{ }^{\circ} \mathrm{C}$ over prolonged periods of time (step IV, highlighted in Fig. 4b) is consistent with our previous reported results. ${ }^{22}$ The disappearance of this signal occurs concomitantly with the dissolution of Se.

Fig. 5 displays FTIR spectra of Se containing species at steps I to IV. The cis and trans isomerization is detected in the case of Se dissolution. As shown in Fig. 5, cis hydrogen out of plane deformation (as highlighted, 'band F', step I) almost disappeared and an increase of trans (band ' $\mathrm{E}$ ') is observed after the total dissolution of Se (step IV). This observation is consistent with the HSQC NMR results shown in Fig. 4 and our previous report which further confirmed the disappearance of the olefinic moiety during the Se dissolution in oleylamine. ${ }^{22}$

Fig. 6 shows the ${ }^{77}$ Se NMR spectrum of the Se precursor generated at step V. Two major signals at $\delta=336.2$ and 525.6 ppm show the characteristic peaks of polyalkyl polyselenides compounds. ${ }^{20}$ This result is similar to our previous findings on Se dissolution into 1-ODE. Moreover, the ${ }^{77}$ Se NMR spectrum shows the presence of a multiplet (highlighted in the inset image, $\delta=336.2 \mathrm{ppm}$ ) indicating the formation of the polyalkyl polyselenides structure.

Fig. 7 displays the $2 \mathrm{D}{ }^{1} \mathrm{H}$ and ${ }^{77} \mathrm{Se} \mathrm{HMBC}$ analysis of the Se precursor in oleylamine. The main ${ }^{77}$ Se signal at $\delta=336.2 \mathrm{ppm}$ correlates with protons at chemical shifts of $\delta=2.81,1.55$ and $1.32 \mathrm{ppm}$. It should be noted that these results are comparable 

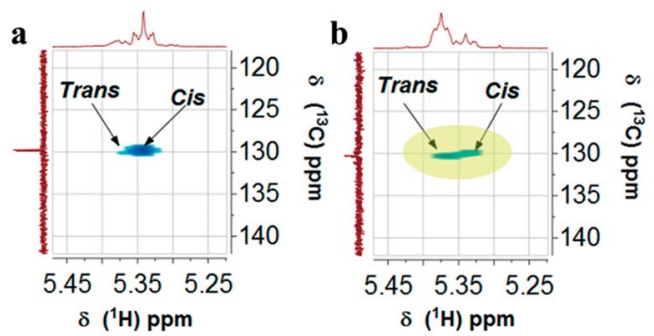

Fig. 4 2D HSQC NMR analysis of (a) Se and oleylamine mixture before heating; (b) Se and oleylamine after heating at $200{ }^{\circ} \mathrm{C}$ for $20 \mathrm{~h}$.

to those in Fig. 3, except for the new signal at $2.81 \mathrm{ppm}$. Moreover, the ${ }^{1} \mathrm{H}$ and ${ }^{13} \mathrm{C}$ HMBC NMR spectra in Fig. S9a (see ESI $[\mathrm{S} 6] \dagger$ ) suggest that the secondary carbon bound to Se is next to carbon atoms with chemical shifts at $\delta=26.79$ and 47.24 ppm. The HSQC spectrum in Fig. S9b $\dagger$ shows these carbon atoms are $\mathrm{CH}$ and $\mathrm{CH}_{2}$ moieties. The analysis also shows that $\mathrm{CH}$ is attached to nitrogen $\left({ }^{1} \mathrm{H}\right.$ at $\delta=2.76$ and ${ }^{13} \mathrm{C} \delta=26.78$ ppm). Therefore, besides structure II and polyalkyl polyselenide structures proposed previously, ${ }^{22}$ structure IV is proposed to be one of the other core structures formed after consumption of the olefin moiety.

The disappearance of the olefin group during Se dissolution in oleylamine can be explained by nucleophilic attack of oleylamine. Besides the double bond, oleylamine also possesses a nucleophilic amine group capable of reacting with $\mathrm{Se}^{23}$ forming $\mathrm{N}$-Se intermediates able to form $\mathrm{N}$-centred radical species. Therefore, the organoselenium structures generated in the oleylamine approach are expected to be more complicated. Based on the NMR and FTIR analyses and previous results, a plausible reaction mechanism for the depletion of the olefin moiety during Se dissolution in oleylamine is proposed in Scheme 2. A radical process is thermally initiated by the homolytic cleavage of the Se-Se bond as highlighted in Scheme 2 . The key step is the attack on the cis allylic proton in oleylamine by Se radicals, leading to the isomerization of the double bond and subsequent $\mathrm{H}$ transfer from the Se intermediate species. ${ }^{21}$ Concomitantly, nucleophilic addition from the amine
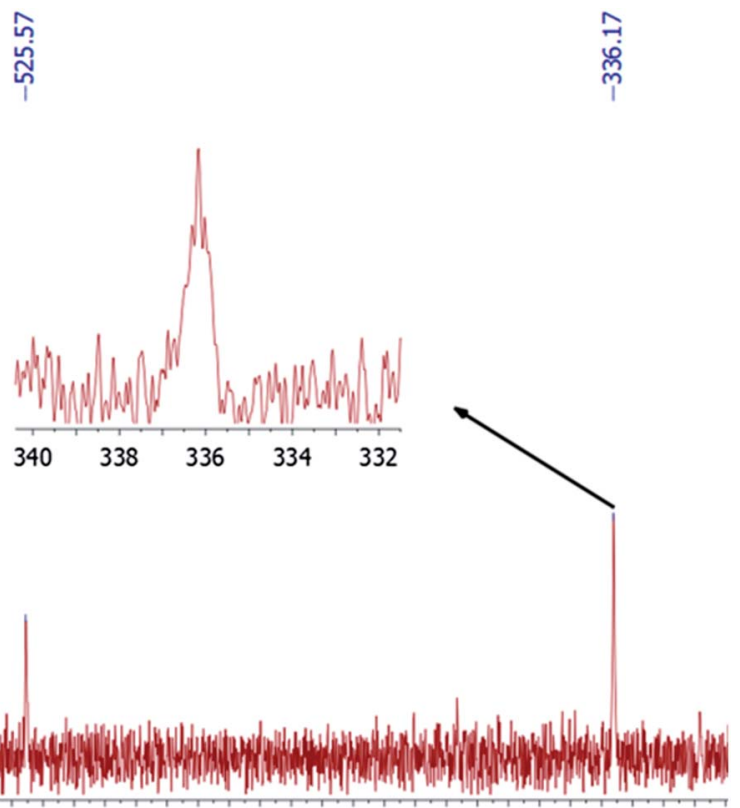

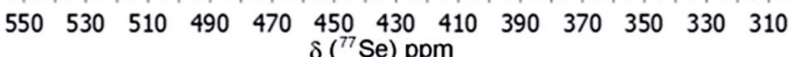

Fig. 6 Proton decoupled ${ }^{77} \mathrm{Se}$ NMR spectrum of the purified Se intermediate generated after dissolution in oleylamine after $20 \mathrm{~h}$ at $200{ }^{\circ} \mathrm{C}$ under $\mathrm{Ar}$. The inset shows the enlarge image of the multiplet peak at $\delta=336.2 \mathrm{ppm}$.

occurs with subsequent addition to the alkyl chain as shown in Scheme 2.

\subsection{Se dissolution into 1-ODE promoted by AIBN}

On the basis that Se solubilisation in 1-ODE occurs via radical species, we investigated whether trace amounts of AIBN could accelerate this process. For instance, recent studies by $\mathrm{Yu}$ et al. have shown that radical cycloaddition reactions involving elemental chalcogens can be accelerated in the presence of AIBN. ${ }^{24}$ Fig. 8 shows clear evidence of migration of the olefin group, and the associated Se dissolution, just after $1 \mathrm{~h}$ heating

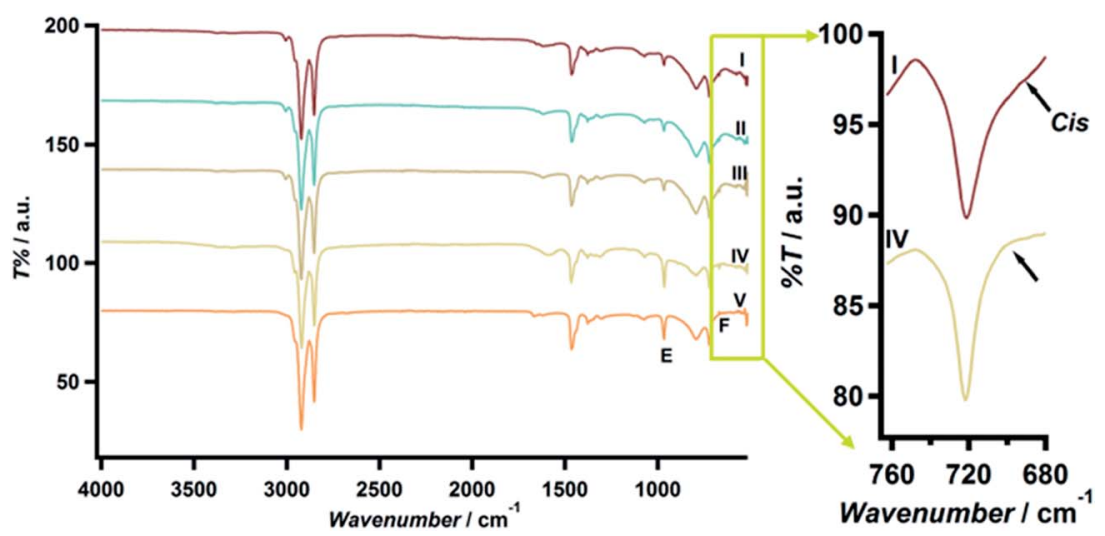

Fig. 5 FTIR analysis of the reaction of oleylamine with Se (I) oleylamine mixed with Se before heating; (II) oleylamine and Se mixture after heating at $60^{\circ} \mathrm{C}$ under vacuum for $30 \mathrm{~min}$; (III) oleylamine and Se after heating at $130^{\circ} \mathrm{C}$ under vacuum for $30 \mathrm{~min}$. (IV) Oleylamine and Se after heating at $200{ }^{\circ} \mathrm{C}$ under Ar for $30 \mathrm{~min}$. (V) Oleylamine and Se after heating at $200^{\circ} \mathrm{C}$ under Ar for $20 \mathrm{~h}$. 


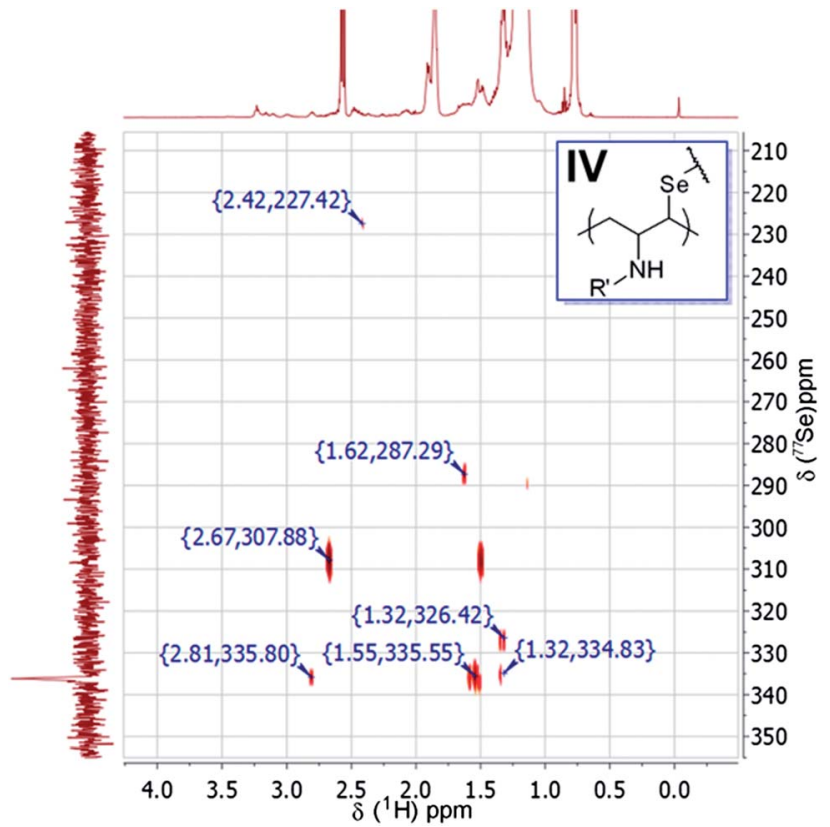

Fig. $7{ }^{1} \mathrm{H}$ and ${ }^{77} \mathrm{Se} \mathrm{HMBC}$ NMR spectra of the Se precursor in oleylamine. As indicated in the image, ${ }^{77} \mathrm{Se}$ (multiplet, $\delta=336.17 \mathrm{ppm}$ ) correlates with protons ${ }^{1} \mathrm{H}$ (multiplet) at $\delta=2.81,1.55$ and $1.32 \mathrm{ppm}$.

$$
\begin{aligned}
& \mathrm{Se}_{\mathrm{n}}-\stackrel{\mathrm{Se}}{-}-\mathrm{Se}-\mathrm{Se}_{\mathrm{n}} \longrightarrow 2 \mathrm{Se}_{\mathrm{n}}-\mathrm{Se} \\
& \mathrm{Se}_{\mathrm{n}}-\mathrm{Se}+\mathrm{H}_{3} \mathrm{C}^{\mathrm{R}} \longrightarrow{ }_{\text {cis }} \mathrm{H}_{2} \mathrm{~N}^{\mathrm{R}} \rightarrow \mathrm{Se}_{\mathrm{n}}-\mathrm{SeH}+\underbrace{\mathrm{R}^{\prime} \mathrm{NH}_{2}}_{\mathrm{R}^{\prime}} \\
& \mathrm{Se}_{n}-\underbrace{\mathrm{Se}-\mathrm{N}_{2}}_{\mathrm{R}}-\mathrm{Se}_{2}-\mathrm{Se}_{n} \rightarrow \mathrm{Se}_{n}-\mathrm{SeH}+\underset{\mathrm{R}-\mathrm{N}-\mathrm{Se}_{\mathrm{H}}-\mathrm{Se}_{\mathrm{n}} \rightarrow \mathrm{R}-\dot{\mathrm{N}} \mathrm{H}}{\mathrm{H}}+\dot{\mathrm{Se}}-\mathrm{Se}_{\mathrm{n}}
\end{aligned}
$$$$
\stackrel{\mathrm{RH}_{2}}{\mathrm{RH}_{\mathrm{R}^{\prime}}^{\mathrm{CH}_{3}}}
$$

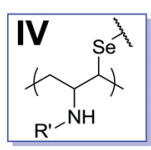

polylalkyl aminoselenides

$R=\leadsto$

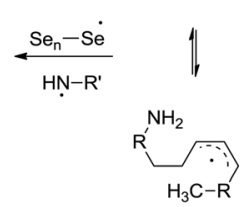

$\mathrm{R}^{\prime}=a \mathrm{lkyl}$, or alkenyl

Scheme 2 Proposed mechanism of the thermal induced Se radical attack on oleylamine, leading to the dissolution of Se and the disappearance of the olefin group.

at $200{ }^{\circ} \mathrm{C}$ in the presence of AIBN. Indeed, the ${ }^{13} \mathrm{C}$ NMR signals at $\delta=124.4$ and $131.7 \mathrm{ppm}$ (Fig. 8a) as well as the ${ }^{1} \mathrm{H}$ NMR multiplet at $\delta=5.42 \mathrm{ppm}$ (Fig. 8b) can be observed after $1 \mathrm{~h}$ as opposed to $20 \mathrm{~h}$ in the absence of AIBN (Fig. 1). The migration of the double bond is further confirmed by HSQC NMR (Fig. 8c and d) as well as FTIR spectroscopy (Fig. 8e), suggesting that AIBN effectively promotes the dissolution of Se. AIBN decomposes at temperatures above $65{ }^{\circ} \mathrm{C}$, eliminating molecular nitrogen and generating 2-cyanoprop-2-yl radicals acting as a

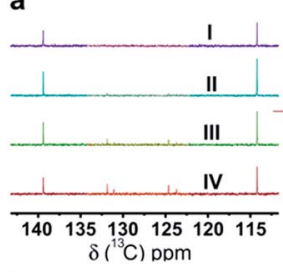

b

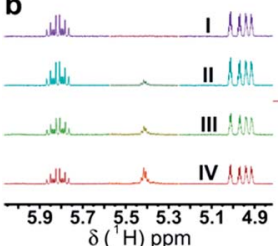

$\delta\left({ }^{\top} \mathrm{H}\right) \mathrm{ppm}$ $c$

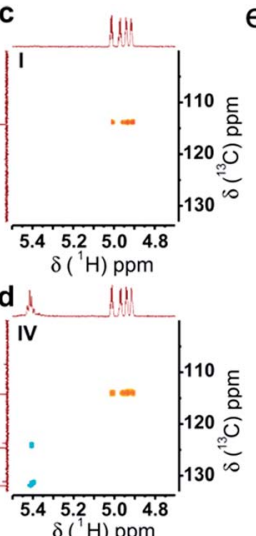

e

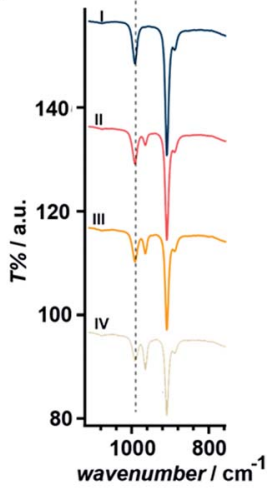

Fig. $8{ }^{13} \mathrm{C}-\mathrm{NMR}(\mathrm{a})$ and ${ }^{1} \mathrm{H}-\mathrm{NMR}(\mathrm{b})$ analyses of the Se-1-ODE mixture at room temperature (step I) and after loading AIBN and heating at 200 ${ }^{\circ} \mathrm{C}$ for 1 (step II), 2 (step III) and $3 \mathrm{~h}$ (step IV) under Ar. HSQC 2D NMR spectra of the mixture at steps I (c) and IV (d). FTIR spectra of the solutions at various stages (e). The main features of the various spectral responses are identical to those highlighted in Fig. 1, although AIBN containing solution promotes Se solubilisation within $1 \mathrm{~h}$.

initiators. ${ }^{25}$ It should be mentioned that, in the absence of Se, the migration of the olefin moiety is not observed when AIBN is present. Consequently, it could be concluded that the radical initiator assists the generation of Se radicals which subsequently attack $\gamma-\mathrm{H}$ in 1-ODE as suggested in Scheme 1.

Reported times for Se dissolution in 1-ODE at various concentrations and temperatures are summarised in Table S2 $(\mathrm{ESI} \dagger)$. The time required for solubilisation of $0.1 \mathrm{~mol} \mathrm{dm}^{-3}$ significantly decreases from $48 \mathrm{~h}$ at $150{ }^{\circ} \mathrm{C}$ to between 1 and $2 \mathrm{~h}$ at $200{ }^{\circ} \mathrm{C}$. Our measurements in the presence of $1 \mathrm{mg}$ of AIBN show slightly shorter times for this Se concentration. The remarkable point is observed at concentrations in the range of $1.0 \mathrm{~mol} \mathrm{dm}^{-3}$, where the dissolution time is decreased from 20 to $3 \mathrm{~h}$ in the presence of AIBN. This temperature is still below the melting point of Se $\left(221{ }^{\circ} \mathrm{C}\right) .^{26}$ Furthermore, the main byproducts of AIBN decomposition are traces of short chain volatile alkanes which do not compromise the purity of the final material.

\subsection{CdSe QD synthesis employing Se precursors generated in the presence of AIBN}

CdSe QDs showing a high degree of monodispersity and strong absorption-luminescence overlap were obtained employing Se precursors in 1-ODE in the presence of AIBN as shown in Fig. 9. The TEM micrograph in Fig. 9a shows highly monodispersed $3.0 \pm 0.2 \mathrm{~nm}$ dots obtained after $15 \mathrm{~min}$ growth time. The inset in Fig. 9a displays absorption and luminescence spectra featuring a strong spectral overlap and a progressive decrease of the optical band gap with increasing growth time.

Selected area electron diffraction pattern rings in Fig. 9b are characteristic of the zinc blende (ZB) unit cell, with the 111, 220 and 311 rings clearly identifiable although broadened by the nanoscopic dimension of the crystals. The experimental data correlate closely with simulations of the diffraction patterns for $3 \mathrm{~nm}$ CdSe nanocrystals. ${ }^{27} \mathrm{X}$-ray powder diffraction (Fig. 9c) is 


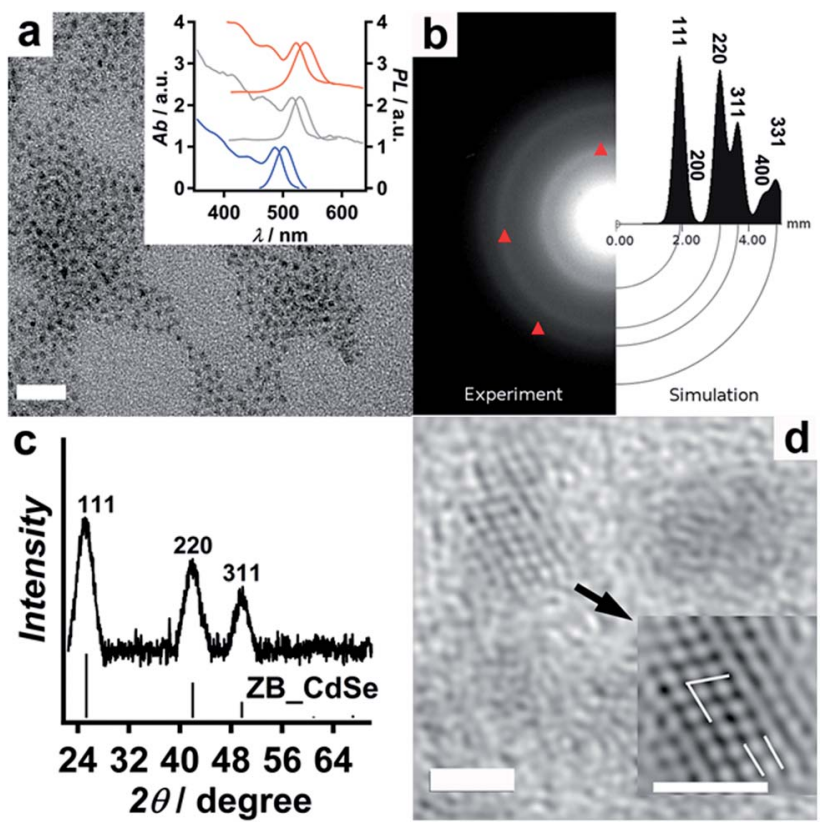

Fig. 9 Characteristic TEM micrograph (scale bar $20 \mathrm{~nm}$ ) of $3.0 \pm 0.2$ $\mathrm{nm}$ CdSe dots after 15 min growth (a). The inset shows the absorption and luminescence spectra recorded after 3 (blue), 8 (grey) and 15 min (red) growth times. Experimental and simulated selected area electron diffraction patterns (b), powder XRD (c) and high resolution TEM image (d, scale bar $2 \mathrm{~nm}$ ) of CdSe dots. The XRD pattern of cubic CdSe (PDF 19-0191) is also shown in (c). The inset in (d) shows a $3.0 \mathrm{~nm}$ dot, highlighting the $70 \pm 2^{\circ}$ cross-grating originating from adjoining (111) planes in fcc crystals with a lattice spacing of $3.5 \pm 0.2 \AA$ (parallel lines).

fully compatible with phase pure ZB CdSe dots. Fig. 9d shows a typical high resolution TEM image obtained from as-prepared 3 nm CdSe QDs, featuring a cross-grating pattern generated by adjoining (111) planes with the $70 \pm 2^{\circ}$ angle. The measured lattice spacing of the (111) plane of $3.5 \pm 0.2 \AA$ is close to the value expected for cubic CdSe (the inset image of Fig. 9d).

These results show that the presence of the trace amount of AIBN does not affect the nucleation of CdSe, demonstrating its suitability for the preparation of Se precursors for QD synthesis. The ability of preparing chalcogenide precursors with high concentration and short times is crucial for deployment of scalable routes for the production of thin-film photovoltaic devices. The introduction of radical initiators in phosphine-free solvents, along with the temperature and concentration, provides an additional optimisation parameter that can be exploited in this direction.

\section{Conclusions}

Our studies have unveiled the processes taking place during the dissolution of Se in 1-ODE and oleylamine, an important bottleneck in the scale-up of phosphine-free synthesis of CdSe QDs. The process is initiated by the generation of Se radicals which attack the allylic proton in both solvents. This reaction manifests itself by the migration of the olefin group in 1-ODE and isomerization in oleylamine.
In the case of 1-ODE, the most plausible intermediates in this process are identified as 1,3-di(octadec-3-en-2-yl)monoselenide, 1,3-di(octadec-3-en-2-yl)diselenide and 1,3-di(octadec-3-en-2-yl)triselenide. On the other hand, the intrinsic nucleophilic properties of oleylamine facilitate the reaction with the double bond during Se solubilisation, inducing the formation of polyalkyl aminoselenides besides the polyalkyl polyselenide species.

For the first time, we show that addition of trace amounts of AIBN significantly accelerates the solubilisation of Se in 1-ODE in the molar concentration range. Studies carried out at various concentrations and temperatures indicate that Se solubilisation requires temperatures above $150{ }^{\circ} \mathrm{C}$, even in the presence of AIBN. Although AIBN promotes faster Se dissolution in the 0.1 mol $\mathrm{dm}^{-3}$ concentration range, the strongest effects are observed at significantly higher Se concentrations.

The utilisation of AIBN as a promoter in the generation of Se precursors allows the facile phosphine-free synthesis of highquality zinc-blende CdSe QDs without the need for further additives. The crystal structure of the dots contrasts with the strong wurtzite character of the materials obtained in the presence of phosphine groups. ${ }^{28}$ We strongly believe that optimization of the chalcogenide precursor's composition by adjusting the concentration of radical initiators, such as AIBN, can have a significant impact on scaling up the synthesis of these materials and their deployment in solution-processable photovoltaic technologies.

\section{Acknowledgements}

The authors are grateful to Mr Jonathan Jones, Mr Paul Lawrence and Dr Craig Butts for their support to this research. This research is supported by the Engineering and Physical Sciences Research Council (EP/G031088/1, SUPERGEN Consortium on Excitonic Solar Cells (DJF and BH) and EP/J002542/1 (MCG and DBA)).

\section{References}

1 Y. Shirasaki, G. J. Supran, M. G. Bawendi and V. Bulovic, Nat. Photonics, 2013, 7, 13-23.

2 (a) F. A. Esteve-Turrillas and A. Abad-Fuentes, Biosens. Bioelectron., 2013, 41, 12-29; (b) F. Wang, X. Liu and I. Willner, Adv. Mater., 2013, 25, 349-377; (c) X. Michalet, F. F. Pinaud, L. A. Bentolila, J. M. Tsay, S. Doose, J. J. Li, G. Sundaresan, A. M. Wu, S. S. Gambhir and S. Weiss, Science, 2005, 307, 538-544.

3 (a) L. Etgar, Materials, 2013, 6, 445-459; (b) P. V. Kamat, J. Phys. Chem. C, 2008, 112, 18737-18753.

4 (a) T. Trindade, P. O'Brien and N. L. Pickett, Chem. Mater., 2001, 13, 3843-3858; (b) C. B. Murray, D. J. Norris and M. G. Bawendi, J. Am. Chem. Soc., 1993, 115, 8706-8715.

5 (a) Z. A. Peng and X. Peng, J. Am. Chem. Soc., 2000, 123, 183184; (b) L. Qu, Z. A. Peng and X. Peng, Nano Lett., 2001, 1, 333-337. 
6 (a) C. de Mello Donegá, P. Liljeroth and D. Vanmaekelbergh, Small, 2005, 1, 1152-1162; (b) K. L. Sowers, B. Swartz and T. D. Krauss, Chem. Mater., 2013, 25, 1351-1362.

7 B. Stewart, A. Harriman and L. J. Higham, Organometallics, 2011, 30, 5338-5343.

8 J. Jasieniak, C. Bullen, J. van Embden and P. Mulvaney, J. Phys. Chem. B, 2005, 109, 20665-20668.

9 (a) H. Shen, H. Wang, Z. Tang, J. Z. Niu, S. Lou, Z. Du and L. S. Li, CrystEngComm, 2009, 11, 1733-1738; (b) M. Sun and X. Yang, J. Phys. Chem. C, 2009, 113, 8701-8709; (c) L. Liu, Z. Zhuang, T. Xie, Y.-G. Wang, J. Li, Q. Peng and Y. Li, J. Am. Chem. Soc., 2009, 131, 16423-16429.

10 S. Sapra, A. L. Rogach and J. Feldmann, J. Mater. Chem., 2006, 16, 3391-3395.

11 (a) Z. Deng, H. Yan and Y. Liu, J. Am. Chem. Soc., 2009, 131, 17744-17745; (b) B. Xing, W. Li, X. Wang, H. Dou, L. Wang, K. Sun, X. He, J. Han, H. Xiao, J. Miao and Y. Li, J. Mater. Chem., 2010, 20, 5664-5674.

12 (a) S. Mourdikoudis and L. M. Liz-Marzan, Chem. Mater., 2013, 25, 1465-1476; (b) Y. Wei, J. Yang, A. W. H. Lin and J. Y. Ying, Chem. Mater., 2010, 22, 5672-5677.

13 S. C. Riha, B. A. Parkinson and A. L. Prieto, J. Am. Chem. Soc., 2011, 133, 15272-15275.

14 Y. Liu, D. Yao, L. Shen, H. Zhang, X. Zhang and B. Yang, J. Am. Chem. Soc., 2012, 134, 7207-7210.

15 (a) S. Flamee, M. Cirillo, S. Abe, K. De Nolf, R. Gomes, T. Aubert and Z. Hens, Chem. Mater., 2013, 25, 2476-2483; (b) S. Flamee, R. Dierick, M. Cirillo, D. Van Genechten, T. Aubert and Z. Hens, Dalton Trans., 2013, 42, 12654-12661. 16 M. Ibanez, R. Zamani, A. LaLonde, D. Cadavid, W. Li, A. Shavel, J. Arbiol, J. R. Morante, S. Gorsse, G. J. Snyder and A. Cabot, J. Am. Chem. Soc., 2012, 134, 4060-4063.
17 (a) C. Bullen, J. van Embden, J. Jasieniak, J. E. Cosgriff, R. J. Mulder, E. Rizzardo, M. Gu and C. L. Raston, Chem. Mater., 2010, 22, 4135-4143; (b) R. García-Rodríguez, M. P. Hendricks, B. M. Cossairt, H. Liu and J. S. Owen, Chem. Mater., 2013, 25, 1233-1249.

18 W. W. Yu and X. Peng, Angew. Chem., Int. Ed., 2002, 41, 23682371.

19 J. D. Odom, W. H. Dawson and P. D. Ellis, J. Am. Chem. Soc., 1979, 101, 5815-5822.

20 H. Eggert, O. Nielsen and L. Henriksen, J. Am. Chem. Soc., 1986, 108, 1725-1730.

21 R. M. Hoyte and D. B. Denney, J. Org. Chem., 1974, 39, 26072612.

22 B. Hou, D. Benito-Alifonso, N. Kattan, D. Cherns, M. C. Galan and D. J. Fermín, Chem. - Eur. J., 2013, 19, 15847-15851.

23 J. Lu, Y. Xie, F. Xu and L. Zhu, J. Mater. Chem., 2002, 12, 2755-2761.

24 L. Yu, Y. Wu, T. Chen, Y. Pan and Q. Xu, Org. Lett., 2012, 15, 144-147.

25 M. B. Smith and J. March, March's Advanced Organic Chemistry: Reactions, Mechanisms, and Structure, WILEY, 6th edn, 2007.

26 Y. A. Yang, H. Wu, K. R. Williams and Y. C. Cao, Angew. Chem., Int. Ed., 2005, 44, 6712-6715.

27 P. A. Stadelmann, Ultramicroscopy, 1987, 21, 131-145.

28 (a) W. W. Yu, Y. A. Wang and X. Peng, Chem. Mater., 2003, 15, 4300-4308; (b) B. Hou, D. Parker, G. P. Kissling, J. A. Jones, D. Cherns and D. J. Fermín, J. Phys. Chem. C, 2013, 117, 6814-6820; (c) Y. C. Li, H. Z. Zhong, R. Li, Y. Zhou, C. H. Yang and Y. F. Li, Adv. Funct. Mater., 2006, 16, 17051716. 